\title{
Recent developments in limited stage small cell lung cancer
}

\author{
Kristin A. Higgins ${ }^{1}$, Sophia Gorgens ${ }^{1}$, Lisa J. Sudmeier ${ }^{1}$, Corinne Faivre-Finn ${ }^{2}$ \\ ${ }^{1}$ Department of Radiation Oncology, Winship Cancer Institute of Emory University, Atlanta, GA, USA; ${ }^{2}$ Department of Thoracic Oncology, \\ University of Manchester, The Christie NHS Foundation Trust, Withington, Manchester, UK \\ Contributions: (I) Conception and design: KA Higgins, C Faivre-Finn; (II) Administrative support: None; (III) Provision of study materials or patients: \\ KA Higgins, C Faivre-Finn; (IV) Collection and assembly of data: All authors; (V) Data analysis and interpretation: All authors; (VI) Manuscript \\ writing: All authors; (VII) Final approval of manuscript: All authors. \\ Correspondence to: Dr. Kristin A. Higgins. 1365 Clifton RD NE, Atlanta, GA 30322, USA. Email: kristin.higgins@emory.edu.
}

\begin{abstract}
Limited stage small cell lung cancer (LS-SCLC) remains a challenging disease, with 5 -year overall survival ranging from 30-35\% with current standard of care treatment consisting of thoracic radiation to $45 \mathrm{~Gy}$ in 30 fractions delivered twice daily, with concurrent platinum/etoposide chemotherapy, followed by prophylactic cranial irradiation (PCI). The randomized, phase III CONVERT study confirmed 45 Gy delivered twice daily to be the optimal radiation fractionation regimen, without significantly increased toxicity when compared to daily radiation to 66 Gy. Immunotherapy is now being studied in addition to chemoradiation, in both the concurrent and consolidative setting. These randomized trials are ongoing. Additionally, the role of PCI compared to MRI surveillance is being evaluated in patients with LS-SCLC in both the North America and Europe. Ideally these ongoing studies will continue to improve outcomes for LS-SCLC.
\end{abstract}

Keywords: Limited stage; small cell lung cancer (SCLC); immunotherapy; prophylactic cranial irradiation (PCI)

Submitted May 07, 2019. Accepted for publication May 15, 2018.

doi: $10.21037 /$ tlcr.2019.05.13

View this article at: http://dx.doi.org/10.21037/tlcr.2019.05.13

\section{Introduction}

Small cell lung cancer (SCLC), while comprising only $13 \%$ of all lung cancers (1), remains difficult to treat with hallmarks including initial high response rates followed by progression of disease that is often recalcitrant to further treatment. Staging is paramount in the prognosis of smallcell lung cancer, with historic staging systems defining limited stage SCLC (LS-SCLC) as disease confined to one hemi-thorax that could safely be encompassed in a single radiation portal (2). The International Association for the Study of Lung Cancer recommended in 2009 the use of the TNM classification for the staging of SCLC based on analysis of 8,088 cases (3).

LS-SCLC has modest 5 -year survival rates of approximately $30-35 \%$ in recent clinical trials (4). Recent developments in the treatment of LS-SCLC include publication of the CONVERT trial, demonstrating that 45 Gy in 30 fractions thoracic radiation delivered twice daily with concurrent chemotherapy remains the standard of care compared with daily fractionated chemoradiation to 66 Gy in 33 fractions, with similar and tolerable toxicity profiles (4). Additionally, there are several current clinical trials underway that offer novel treatment paradigms for LS-SCLC. Trials are ongoing that combine immune check point inhibitors with chemoradiation, and the role of prophylactic cranial irradiation (PCI) also continues to be refined. This article reviews the standard of care for LSSCLC as well as recent developments in the treatment of LS-SCLC.

\section{Standard of care treatment is $45 \mathrm{~Gy}$ bid with concurrent platinum/etoposide + PCI}

The current standard of care in patients with LS-SCLC is based on a randomised controlled trial that compared once-daily (OD-45 Gy in 25 fractions over 5 weeks) to twice-daily (BID; 45 Gy in 30 fractions over 3 weeks) 
radiotherapy (RT) delivered concurrently with cisplatin and etoposide showing the superiority of BID RT in terms of survival (5). However since this publication in 1999, there has been a lack of consensus regarding the routine use of BID RT, despite its superiority, due to concerns regarding toxicity (i.e., one third of the patients developing $\geq$ grade 3 radiation oesophagitis) and logistical issues. International guidelines highlighted the need for further trials in this group of patients which led to the development of the CONVERT trial (4) comparing BID RT (45 Gy in 30 fractions over 3 weeks) to a higher dose of RT delivered OD (66 Gy in 33 fractions over 6.5 weeks), both given concurrently with chemotherapy (staring with cycle 2). CONVERT is the largest study ever completed investigating chemoradiotherapy in SCLC cancer and is the first randomised trial providing outcome data in patients staged with positron emission tomography-computed tomography (PET-CT), using the TNM classification and treated with modern RT techniques (i.e., 3D conformal RT or Intensity Modulated RT, no elective nodal irradiation). CONVERT recruited 547 patients in 8 countries and incorporated a RT quality assurance programme $(6,7)$.

Overall survival outcomes did not significantly differ between the two groups (hazard ratio for death in the once daily group at median follow up of 45 months $=1.18$, $\mathrm{P}=0.14$ ), however survival achieved in both groups is higher and toxicity much lower ( $>50 \%$ reduction) than previously reported in the literature. At a median follow-up of 45 months, 2 -year overall survival was $56 \%$ in the BID group and $51 \%$ in the OD group and 5-year survival 34\% in the BID group and $31 \%$ in the OD group. There was no difference in grade 3-4 oesophagitis between the groups $19 \%$ in the BID group vs. $19 \%$ in the OD group and grade $3-4$ radiation pneumonitis, $3 \%$ in the BID vs. $2 \%$ in the OD group. Since the trial was designed to show superiority of OD RT and was not powered to show equivalence, the implication is that BID RT should continue to be considered the standard of care in this group of patients. The CONVERT trial results will help to standardize patient care which is currently very variable (8). Importantly it demonstrates that in the era of modern RT techniques, the frequency and severity of acute and late radiation toxicities are lower than previously reported.

Furthermore, CONVERT has established a unique dataset which has provided an opportunity to investigate many aspects of this disease in subgroup analyses. Given the lack of data on the efficacy and safety of concurrent chemoradiotherapy in elderly, the CONVERT team compared the outcome of patients aged $\geq 70$ years $v$ s. younger patients (9). Fewer older patients received the optimal number of RT fractions ( $73 \%$ vs. $85 \%$ ); however, chemotherapy compliance was similar in both groups. There was more haematological toxicity (neutropenia) in the elderly group but the rates of radiation pneumonitis and esophagitis were similar. Therefore it can be concluded that concurrent chemoradiotherapy with modern RT techniques is a treatment option for fit, older patients. CONVERT has also provided outcome data, for the first time in the context of a randomised trial, on patients staged using the TNM classification $(10,11)$. TNM stage I-II SCLC patients (87 patients) were compared to stage III (422 patients). Stage I-II patients achieved longer OS [median: 50 (95\% CI, 38-not reached) vs. 25 (95\% CI, 21-29) months; $\mathrm{P}=0.001]$ compared to stage III. Apart from lower incidence of acute esophagitis in stage I-II compared to stage III patients (grade $\geq 3: 11 \%$ vs. $21 \% ; \mathrm{P}<0.001$ ), the incidence of acute and late toxicities was not significantly different (10). This study therefore benchmarks the outcome of stage I-II SCLC patients treated with modern chemoradiotherapy, providing information that clinicians can relay to their patients to aid clinical decisions (such as surgery $v s$. chemoradiotherapy).

Finally, the impact of ${ }^{18} \mathrm{~F}$-fludeoxyglucose $\left({ }^{18} \mathrm{~F}\right.$-FDG $)$ $\mathrm{PET} / \mathrm{CT}$ in SCLC management was investigated as part of the CONVERT study (12). ${ }^{18}$ F-FDG PET/CT staging was allowed but not mandated. The outcome of patients staged with conventional imaging (contrast-enhanced thorax and abdomen CT and brain imaging with/without bone scintigraphy) and those staged with ${ }^{18} \mathrm{~F}-\mathrm{FDG}$ PET/ $\mathrm{CT}$ in addition was compared. There were no significant differences in overall (HR 0.87, 95\% CI, 0.70-1.08; $\mathrm{P}=0.192$ ) and progression-free survival (HR 0.87, 95\% CI, 0.71-1.07; $\mathrm{P}=0.198)$ between patients staged with or without ${ }^{18} \mathrm{~F}-\mathrm{FDG}$ PET/CT. These findings could guide the selection of LS-SCLC patients for treatment with concurrent chemoradiotherapy. However, this analysis cannot support the use or omission of ${ }^{18} \mathrm{~F}$-FDG PET/CT due to unplanned nature of this subgroup analysis.

With regards to further studies, a number of ideas have been generated since the CONVERT results were presented including; dose escalation of the BID arm of CONVERT, the investigation of hypofractionated RT and the stratification of patients based on CTC/cfDNA to validate the findings of our preliminary translational studies (13). The aim would be to identify patients who may not benefit from concurrent chemoradiotherapy or may need further 
consolidation systemic therapy.

\section{Refining PCI}

PCI confers an overall survival benefit in patients with LSSCLC and is considered standard of care (14). In a 1999 meta-analysis, Aupérin and colleagues analyzed data on individual data of 987 patients with SCLC in complete remission who had been enrolled in seven clinical trials comparing PCI vs. no PCI. Their results demonstrated a $5.4 \%$ absolute improvement in three-year overall survival (20.7\% vs. $15.3 \%$ ), improved disease-free survival (relative risk $=0.75, \mathrm{P}<0.001$ ), and decreased incidence of brain metastases (relative risk $=0.46, \mathrm{P}<0.001$ ) with the addition of PCI (15). Given this benefit, PCI is standard therapy for all patients with LS-SCLC and is a component of the treatment paradigm for LS-SCLC patients enrolled on randomized trials. In a recent survey of U.S. radiation oncologists, 98\% recommended PCI for patients with LSSCLC (16). There are, however, certain populations for which PCI should be withheld, including patients that immediately progress after definitive therapy for LS-SCLC, and patients with baseline neurocognitive disabilities, however the vast majority of patients with LS-SCLC should be offered PCI as part of standard of care treatment. It should however be noted that the role of PCI is not as well defined in patients with stage I-II SCLC.

The role of PCI in ES-SCLC has evolved over time and is pertinent to this review as ongoing clinical trials are assessing PCI in both the ES-SCLC and LS-SCLC populations collectively. In 2007, an EORTC trial showed that PCI improved overall survival in patients with ESSCLC that derived any response from first-line systemic therapy (17). Thus, PCI became the standard of care in the ES-SCLC setting for a number of years; however, a Japanese randomized trial recently showed that PCI did not increase overall survival for patients with ES-SCLC (18). In the Japanese trial, patients were re-staged with MRI of the brain and then randomized to either PCI or observation, with MRI of the brain performed every 3 months. At a planned interim analysis in 2013, the study was terminated early due to futility. Median overall survivals were 11.6 and 13.7 months for the PCI and observation groups respectively (18). Given the most recent Japanese study, practice patterns in the U.S. have shifted to the majority of radiation oncologists not recommending PCI in the ES-SCLC setting (19). In contrast a consensus analysis of group of 13 European experts from the European Society for Therapeutic Radiation Oncology and the International Association for the Study of Lung Cancer was performed. It showed that European radiation and medical oncologists specializing in lung cancer recommend PCI in selected patients and restrict its use primarily to fit, non-elderly patients who responded to chemotherapy (20).

One trial investigating PCI in both LS-SCLC and ESSCLC is the currently ongoing NRG CC003 randomized phase II/III trial, which utilizes hippocampal sparing as an approach to potentially reduce neurocognitive toxicity (ClinicalTrials.gov Identifier: NCT02635009). As the hippocampus is involved in memory, the investigators hypothesize that sparing the hippocampus with intensity modulated radiation therapy or volumetric arc therapy may decrease cognitive side-effects, particularly memory, typically seen in PCI. The trial started enrolling in late 2015 and hopes to have a primary completion date in 2022 with a study completion date in 2027 . The primary outcomes will be HVLT-R delayed recall deterioration status (phase III primary endpoint) and intracranial relapse rate (phase II primary endpoint), and will include overall survival as a secondary endpoint.

Lastly, a Southwest Oncology Group study will evaluate the role of PCI versus MRI surveillance every 3 months in patients with both limited and extensive stage SCLC. This study will stratify patients based on LS-SCLC $v s$. ESSCLC, usage of immunotherapy, and performance status, with a primary endpoint of overall survival. A similar study is in set up within the EORTC as well.

\section{Chemoradiation and immunotherapy}

There has recently been a major development in the treatment of ES-SCLC as the IMpower133 study has demonstrated improvements in progression free and overall survival when atezolizumab, a PD-L1 inhibitor, was added to first-line chemotherapy and continued into the maintenance phase (21). Median progression-free survival for the atezolizumab group was 5.2 and 4.3 months for the control group (21). Atezolizumab is now FDA approved for use in the first-line setting for patients with ES-SCLC. This is a major advancement for ES-SCLC given no new drugs have shown improvements in survival in this patient population in several decades.

Following on from positive trials adding immunotherapy to standard of care treatment in ES-SCLC and in stage III NSCLC (22), an ongoing question is whether immunotherapy could improve survival in the limited stage 
setting. The rationale for immunotherapy concurrent with chemoradiation has been shown in a multitude of preclinical studies, including xenograft models of pancreatic, colon, and breast cancer $(23,24)$. Radiation to a primary tumor leads to tumor-antigen release and a tumor specific adaptive immune response, which is enhanced by immune-stimulating agents. Thus, while radiation induces a local tumor response at the site of radiation, it may also lead to regression of micrometastatic disease (the abscopal effect) (25). Additional preclinical data supporting the use of immune check point inhibitors and radiation concurrently include a study showing that low doses of fractionated radiation given concurrently with anti PD-1 antibodies enhanced local tumor control and elicited out of field responses in a mouse model of colon cancer, with a $>70 \%$ complete response rate (26). Sequential delivery of checkpoint inhibitors did not improve response to RT at local or distant sites. Next generation sequencing of $\mathrm{T}$ cell receptors showed that while radiation increased $\mathrm{T}$ cell infiltration at the irradiated tumor only, radiation + anti PD-1 increased $\mathrm{T}$ cell infiltration/expansion at both irradiated and out-of-field tumors. Additionally, radiation + anti-PD-1 increased T-cell receptor diversity more than radiation or anti $\mathrm{PD}-1$ alone (26).

There are three randomized trials currently investigating the addition of immunotherapy to chemoradiotherapy in LS-SCLC. NRG Oncology and Alliance are currently testing the use of atezolizumab after chemoradiotherapy in the LS-SCLC setting (NCT03811002). This is a phase II/III trial comparing standard chemoradiation alone to atezolizumab plus concurrent chemoradiation, with atezolizumab and thoracic radiation beginning with the second cycle of chemotherapy. Atezolizumab is given every 3 weeks concurrent with radiation and continuing on for a total of 12 months. Stratification variables include performance status (PS 0/1 vs. 2), sex, use of chemotherapy (cisplatin $v s$. carboplatin), and radiation fractionation (twice daily to 45 Gy versus once daily to 66 Gy). PCI (25 Gy in 10 fractions) is recommended in patients that have a complete or near complete response to therapy. The primary endpoint of the phase II component is PFS and the primary endpoint of the phase III component is OS. Secondary endpoints include immune and non-immune related toxicities, quality of life, distant-metastases free survival, local control and overall response rates. This study opened to accrual in May of 2019 (ClinicalTrials.gov
Identifier: NCT03811002).

Another trial utilizing immunotherapy for the treatment of LS-SCLC is the European Thoracic Oncology Platform (ETOP) sponsored STIMULI trial which is a phase II randomized trial investigating if the use of consolidation with nivolumab and ipilimumab in LSSCLC after chemoradiation therapy and PCI is better than chemoradiation therapy and PCI alone (ClinicalTrials. gov Identifier: NCT02046733). Nivolumab is a humanized monoclonal antibody that targets PD-1 while and ipilimumab targets CTLA-4. The primary endpoints are progression-free and overall survival (9). The study closed early in 2019 due to slow accrual.

Another clinical trial testing the role of immunotherapy is the phase III, randomized, double-blind, international study ADRIATIC study sponsored by AstraZeneca (ClinicalTrials.gov Identifier: NCT03703297). This is a three-arm studying evaluating the efficacy of Durvalumab or Durvalumab with Tremelimumab compared to placebo for consolidation in LS-SCLC patients who have not progressed after concurrent chemoradiation. Durvalumab blocks PD-L1 and prevents it from binding to PD-1 and CD80, while Tremelimumab blocks CTLA-4 and prevents it from binding to ligands on antigen-presenting cells. In a phase Ib study with non-small cell lung cancer patients, the combination of durvalumab with tremelimumab showed clinical antitumor activity regardless of PD-L1 expression (27). The primary endpoints of this phase III study are progression-free survival and overall survival.

Table 1 below summarizes the active clinical trials that are evaluating immunotherapy in patients with LS-SCLC.

\section{Conclusions}

While LS-SCLC remains a therapeutic challenge, the last decade has seen progress for this patient population. The pivotal CONVERT study has confirmed 45 Gy delivered twice daily with chemotherapy as the standard of care for this population, without significant toxicity relative to daily radiation. Additionally, several ongoing studies are evaluating the role of immunotherapy in combination with chemoradiation. Lastly, PCI techniques with hippocampal avoidance are being examined in LS-SCLC, along with the role of MRI surveillance. Ideally, these therapeutic strategies will continue to improve long-term survival and quality of life for patients with LS-SCLC. 
Table 1 Ongoing studies evaluating immunotherapy in LS-SCLC patients

\begin{tabular}{|c|c|c|c|c|c|}
\hline $\begin{array}{l}\text { Study name } \\
\text { (start date) }\end{array}$ & Phase & Arm I & Arm II & Arm III & $\begin{array}{l}\text { Primary } \\
\text { endpoints }\end{array}$ \\
\hline NRG LU005 & II/III & $\begin{array}{l}\text { ChemoRT: etoposide }+ \\
\text { cisplatin or carboplatin q } 21 \\
\text { days } \times 3 \text { cycles with either } \\
\text { BID or daily radiation therapy }\end{array}$ & $\begin{array}{l}\text { ChemoRT + IO: treatment as in } \\
\text { Arm I + atezolizumab IV on day } \\
1 \text { or } 2 \text { of each chemo cycle and } \\
\text { then every } 3 \text { weeks } \times 17 \text { cycles }\end{array}$ & $\mathrm{N} / \mathrm{A}$ & PFS, OS \\
\hline ADRIATIC & III & $\begin{array}{l}\text { ChemoRT + IO: ChemoRT } \\
\text { as in Arm III followed by } \\
\text { Durvalumab and placebo } \\
\text { saline solution IV q4 weeks } \\
\times 4 \text { followed by durvalumab } \\
\text { monotherapy q4 weeks }\end{array}$ & $\begin{array}{l}\text { ChemoRT + IO: ChemoRT as in } \\
\text { Arm III followed by durvalumab } \\
\text { and tremelimumab IV q4 weeks } \\
\times 4 \text { followed by durvalumab } \\
\text { monotherapy q4 weeks }\end{array}$ & $\begin{array}{l}\text { ChemoRT: etoposide }+ \\
\text { platinum-based chemo q } 21 \\
\text { days } \times 4 \text { cycles with either BID } \\
\text { or daily radiation therapy then } \\
\text { two placebo saline solutions } \\
\text { (IV) q4 week } \times 4 \text {, followed } \\
\text { by placebo saline solution } \\
\text { monotherapy q4 weeks }\end{array}$ & PFS, OS \\
\hline
\end{tabular}

LS-SCLC, limited stage small cell lung cancer; ChemoRT, concurrent chemotherapy and radiation therapy; IO, immuno-oncology; PFS, progression-free survival; OS, overall survival; N/A, not applicable.

\section{Acknowledgments}

Funded research: RefleXion Medical.

\section{Footnote}

Conflicts of Interest: Dr. KA Higgins's disclosures: consultant: Astra Zeneca, Varian. Advisory board: Genentech, Astra Zeneca. The other authors have no conflicts of interest to declare.

\section{References}

1. Govindan R, Page N, Morgensztern D, et al. Changing epidemiology of small-cell lung cancer in the United States over the last 30 years: analysis of the surveillance, epidemiologic, and end results database. J Clin Oncol 2006;24:4539-44.

2. Zelen M. Keynote address on biostatistics and data retrieval. Cancer Chemother Rep 3 1973;4:31-42.

3. Vallieres E, Shepherd FA, Crowley J, et al. The IASLC Lung Cancer Staging Project: proposals regarding the relevance of TNM in the pathologic staging of small cell lung cancer in the forthcoming (seventh) edition of the TNM classification for lung cancer. J Thorac Oncol 2009;4:1049-59.

4. Faivre-Finn C, Snee M, Ashcroft L, et al. Concurrent once-daily versus twice-daily chemoradiotherapy in patients with limited-stage small-cell lung cancer (CONVERT): an open-label, phase 3, randomised, superiority trial. Lancet Oncol 2017;18:1116-25.

5. Turrisi AT 3rd, Kim K, Blum R, et al. Twice-daily compared with once-daily thoracic radiotherapy in limited small-cell lung cancer treated concurrently with cisplatin and etoposide. N Engl J Med 1999;340:265-71.

6. Groom N, Wilson E, Lyn E, et al. Is pre-trial quality assurance necessary? Experiences of the CONVERT Phase III randomized trial for good performance status patients with limited-stage small-cell lung cancer. $\mathrm{Br} \mathrm{J}$ Radiol 2014;87:20130653.

7. Groom N, Wilson E, Faivre-Finn C. Effect of accurate heart delineation on cardiac dose during the CONVERT trial. Br J Radiol 2017;90:20170036.

8. Komaki R, Khalid N, Langer CJ, et al. Penetration of recommended procedures for lung cancer staging and management in the United States over 10 years: a quality research in radiation oncology survey. Int J Radiat Oncol Biol Phys 2013;85:1082-9.

9. Christodoulou M, Blackhall F, Mistry H, et al. Compliance and Outcome of Elderly Patients Treated in the Concurrent Once-Daily Versus Twice-Daily Radiotherapy (CONVERT) Trial. J Thorac Oncol 2019;14:63-71.

10. Salem A, Mistry H, Hatton M, et al. Association of 
Chemoradiotherapy With Outcomes Among Patients With Stage I to II vs Stage III Small Cell Lung Cancer: Secondary Analysis of a Randomized Clinical Trial. JAMA Oncol 2019;5:e185335.

11. Shepherd FA, Crowley J, Van Houtte P, et al. The International Association for the Study of Lung Cancer lung cancer staging project: proposals regarding the clinical staging of small cell lung cancer in the forthcoming (seventh) edition of the tumor, node, metastasis classification for lung cancer. J Thorac Oncol 2007;2:1067-77.

12. Manoharan P, Salem A, Mistry H, et al. [18F] fludeoxyglucose PET/CT in small-cell lung cancer: Analysis of the CONVERT randomized controlled trial. J Thorac Oncol 2019. [Epub ahead of print].

13. Tay RY, Fernandez-Gutierrez F, Foy V, et al. Prognostic value of circulating tumour cells in limited-stage small cell lung cancer: analysis of the concurrent ONce-daily versus twice-daily RadioTherapy (CONVERT) randomised controlled trial. Ann Oncol 2019. [Epub ahead of print].

14. Rosen ST, Makuch RW, Lichter AS, et al. Role of prophylactic cranial irradiation in prevention of central nervous system metastases in small cell lung cancer. Potential benefit restricted to patients with complete response. Am J Med 1983;74:615-24.

15. Aupérin A, Arriagada R, Pignon JP, et al. Prophylactic cranial irradiation for patients with small-cell lung cancer in complete remission. Prophylactic Cranial Irradiation Overview Collaborative Group. N Engl J Med 1999;341:476-84.

16. Farrell MJ, Yahya JB, Degnin C, et al. Prophylactic Cranial Irradiation for Limited-Stage Small-Cell Lung Cancer: Survey of US Radiation Oncologists on Current Practice Patterns. Clin Lung Cancer 2018;19:371-6.

17. Slotman B, Faivre-Finn C, Kramer G, et al. Prophylactic cranial irradiation in extensive small-cell lung cancer. $\mathrm{N}$ Engl J Med 2007;357:664-72.

18. Takahashi T, Yamanaka T, Seto T, et al. Prophylactic cranial irradiation versus observation in patients with extensive-disease small-cell lung cancer: a multicentre,

Cite this article as: Higgins KA, Gorgens S, Sudmeier LJ, Faivre-Finn C. Recent developments in limited stage small cell lung cancer. Transl Lung Cancer Res 2019;8(Suppl 2):S147-S152. doi: 10.21037/tlcr.2019.05.13 randomised, open-label, phase 3 trial. Lancet Oncol 2017;18:663-71.

19. Gjyshi O, Ludmir EB, Pezzi TA, et al. The Impact of the Stage III Randomized Trial by Takahashi et al. on the Use of Prophylactic Cranial Irradiation (PCI) in Patients with Extensive-Stage Small-Cell Lung Cancer (ES-SCLC). Multidisciplinary Thoracic Cancers Symposium; March 14, 2019; San Diego, CA, 2019.

20. Putora PM, Glatzer M, Belderbos J, et al. Prophylactic cranial irradiation in stage IV small cell lung cancer: Selection of patients amongst European IASLC and ESTRO experts. Radiother Oncol 2019;133:163-6.

21. Horn L, Mansfield AS, Szczesna A, et al. First-Line Atezolizumab plus Chemotherapy in Extensive-Stage Small-Cell Lung Cancer. N Engl J Med 2018;379:2220-9.

22. Antonia SJ, Villegas A, Daniel D, et al. Overall Survival with Durvalumab after Chemoradiotherapy in Stage III NSCLC. N Engl J Med 2018;379:2342-50.

23. Blanquicett C, Saif MW, Buchsbaum DJ, et al. Antitumor efficacy of capecitabine and celecoxib in irradiated and lead-shielded, contralateral human BxPC-3 pancreatic cancer xenografts: clinical implications of abscopal effects. Clin Cancer Res 2005;11:8773-81.

24. Deng L, Liang H, Burnette B, et al. Radiation and antiPD-L1 antibody combinatorial therapy induces T cellmediated depletion of myeloid-derived suppressor cells and tumor regression. Oncoimmunology 2014;3:e28499.

25. Tang $\mathrm{C}$, Wang $\mathrm{X}$, Soh $\mathrm{H}$, et al. Combining radiation and immunotherapy: a new systemic therapy for solid tumors? Cancer Immunol Res 2014;2:831-8.

26. Dovedi SJ, Cheadle EJ, Popple AL, et al. Fractionated Radiation Therapy Stimulates Antitumor Immunity Mediated by Both Resident and Infiltrating Polyclonal T-cell Populations when Combined with PD-1 Blockade. Clin Cancer Res 2017;23:5514-26.

27. Antonia S, Goldberg SB, Balmanoukian A, et al. Safety and antitumour activity of durvalumab plus tremelimumab in non-small cell lung cancer: a multicentre, phase $1 \mathrm{~b}$ study. Lancet Oncol 2016;17:299-308. 